\title{
A comparison of machine learning classifiers for smartphone-based gait analysis
}

\author{
Rosa Altilio ${ }^{1} \cdot$ Andrea Rossetti $^{1} \cdot$ Qiang Fang ${ }^{2} \cdot$ Xudong Gu$^{3} \cdot$ Massimo Panella ${ }^{1}$ (D)
}

Received: 1 April 2020 / Accepted: 14 December 2020 / Published online: 6 February 2021

(C) The Author(s) 2021

\begin{abstract}
This paper proposes a reliable monitoring scheme that can assist medical specialists in watching over the patient's condition. Although several technologies are traditionally used to acquire motion data of patients, the high costs as well as the large spaces they require make them difficult to be applied in a home context for rehabilitation. A reliable patient monitoring technique, which can automatically record and classify patient movements, is mandatory for a telemedicine protocol. In this paper, a comparison of several state-of-the-art machine learning classifiers is proposed, where stride data are collected by using a smartphone. The main goal is to identify a robust methodology able to assure a suited classification of gait movements, in order to allow the monitoring of patients in time as well as to discriminate among a pathological and physiological gait. Additionally, the advantages of smartphones of being compact, cost-effective and relatively easy to operate make these devices particularly suited for home-based rehabilitation programs.
\end{abstract}

Keywords Gait analysis · Machine learning classifier · Smartphone technology · Wavelet-based feature extraction · Home-based telemedicine

\section{Introduction}

An important field of application for data classification and screening is the one concerning human motion, in particular the one required in gait analysis, defined as

Massimo Panella

massimo.panella@uniroma1.it

Rosa Altilio

rosa.altilio@uniroma1.it

Andrea Rossetti

rossdamc@gmail.com

Qiang Fang

qiangfang@stu.edu.cn

Xudong $\mathrm{Gu}$

jxgxd@hotmail.com

1 Department of Information Engineering, Electronics and Telecommunications (DIET), University of Rome "La Sapienza”, Via Eudossiana 18, 00184 Rome, Italy

2 Department of Biomedical Engineering, College of Engineering, Shantou University, Shantou 515063, China

3 Second Hospital of Jiaxing, Jiaxing 314000, China the systematic study of human walking [1-5]. As far as we know, considerable information can be extracted by analyzing patients' walking because it contains important biometric features. In effect, gait is related to the walker's physical and, sometimes, psychological state [6].

Decision-making for gait analysis could be supported by the use of computational intelligence techniques for automatic determining the status of a patient. For instance, in [7-9] several classification algorithms are evaluated and compared in terms of their ability to discriminate among physiological and pathological gait.

Generally, the most adopted tools for gait analysis are based on high complexity motion capture systems exploiting active or passive markers, electromyography (EMG), dynamometric platforms, and so on [10-14]. Unfortunately, the high cost and the complexity they require make them suitable only for specific clinicians and hospitals.

To ease the monitoring of patients' motion, while keeping the cost low, a lot of researchers focus their attention on IMU sensors [15-17]. Unfortunately, these sensors have severe drift problems, making necessary their usage in parallel with other technologies [18]. To overcome these issues, some studies have analyzed the impact that new technologies based on both visual and non visual systems 
could bring on the related research and application fields [6, 19-21].

In effect, in the last decade, everyday life has been powerfully influenced by technology, like tablets or smartphones ceaselessly connected through mobile networks. Research is increasingly striving towards the exploitation and the evolution of daily experiences by improving and developing new functionality that people could ask for.

A current and quite recent trend of research has seen the investigation of smartphone-based applications for movement monitoring and analysis, as for example considered in [22]. Gait analysis experiments using a smartphone to demonstrate the capability to accurately quantify gait parameters with a sufficient level of consistency have been performed in [23] and in several other works as, for instance, in [24-27]. However, these approaches mainly rely on human experts (i.e., doctors) for the clinical analysis of smartphone data and making a decision accordingly. The feasibility, efficacy and usefulness of machine learning techniques for discriminating automatically the gait movements and for assessing the main extracted features have not been systematically evaluated so far.

In this work, we extend the use of sensors contained in a smartphone to realize a work particularly suited for a future home-based rehabilitation approach. In particular, its advantages of being compact, cost-effective and relatively easy to operate automatically, compared to the other onerous and expensive technologies, make this device particularly suited for this context. As for the biomedical context, a low-cost smartphone-based system could bring great advantages to both diseased people and clinicians, by upgrading the patients' quality of life and reducing the average rehabilitation cost. Despite this approach being interesting for the treatment of many diseases like Parkinson's, multiple sclerosis and Coxarthrosis, we will describe a specific solution tailored to the monitoring of people recovering from a stroke. In effect, post-stroke rehabilitation has been proven to be essential and effective in helping stroke patients to gradually regain part of their body functionality. In particular, gait analysis, which is the standard practice for diagnosis, assessment, monitoring and discussion of diseases that affect gait, is used to detect the walking patterns and posture that are unique for hemiplegic patients at different recovery stages.

We propose a reliable remote monitoring scheme that can assist medical specialist in watching over the patient's condition. A smartphone is used to collect stride data and obtain useful information from these data by means of advanced features extraction methods. The system should be used to assist medical specialists in analyzing the rehabilitation path at range, also when the patient is not in the hospital anymore. In this way, assuming that the approach is inserted in a well-scheduled program of "home" rehabilitation, it will be possible to reduce costs, while improving the patient's life quality and allowing clinicians to evaluate patient's improvements in a safer and faster manner.

The novelty, with respect to state-of-the-art applications, is the combination of the data acquisition and filtering from the device, with data fusion and pattern recognition techniques that provide a correct definition of the gait movements, allowing to monitor the patient in time, as well as discriminate among a pathological and a physiological gait.

The rest of the paper is organized as follows. We introduce the proposed approach in Section 2. The application is ascertained by extensive computer simulations and several benchmark results, which are reported in Section 3 and discussed in Section 4. Finally, our conclusions are drawn in Section 5 .

\section{Methods}

\subsection{Selection and description of patients}

In the experimental process, which will be described in the detail successively, we evaluate the gait of two set of individuals. A group of both healthy people and poststroke patients took part in the experiment. We collected 60 different walking trials through heterogeneous smartphone devices of different manufacturers. Among these 60 records, 25 of them belong to voluntary unhealthy patients from the Rehabilitation Medical Center of the 2nd Hospital of Jiaxing, Zhejiang province, China; the remaining 35 are healthy persons among academic researchers and doctors of the previously cited Medical Center. Additionally, data differ for the length of the recording session: 41 of them (respectively 13 from patients and 28 from healthy people) are recorded in $10 \mathrm{~s}$, the remaining 19 (12 of which are patients) are recorded in $20 \mathrm{~s}$. People are asked to perform a walking in a straight path, without deviation.

All research activities in this study were conducted in accordance with the ethical principles of the Declaration of Helsinki. As it involves human participants, the present study was performed in accordance with the relevant institutional and national guidelines, with informed written consent from all human subjects involved in the study including for publication of the results. However, the study is exempt from the explicit ethics approval of appropriate institutional Committees, as it is mainly focused on the engineering aspects pertaining to the use of specific ICT technologies as well as signal processing and analysis of the related data. All subject anonymity is preserved as identifying information is not included in the manuscript. 


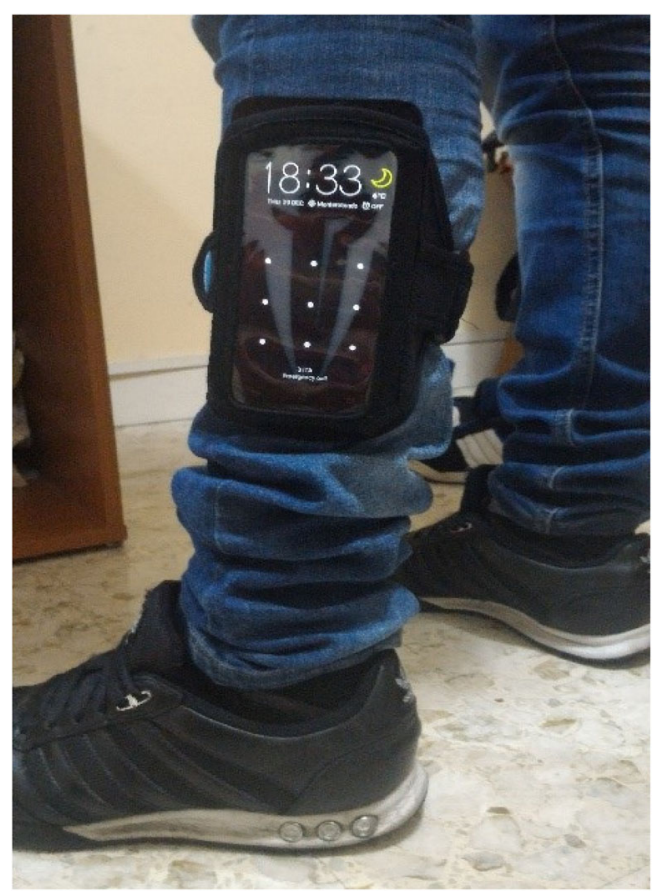

Fig. 1 Position of the smartphone device during the clinical trials

\subsection{Procedural information}

In this study, we perform an analysis of the user's stride in order to extract suitable features for a classification purpose. A low-cost smartphone device (i.e., Samsung's Galaxy A3 2017 SM-A320F with Android v6.0.1) is put in the pocket of a band and ties around the user's calf as shown in Fig. 1. In fact, most of the gait information and lower limb's angle movements are linked to this muscle. One device only is adopted, so as to represent a realistic scenario in a home-based context where a user has one smartphone only.

In order to implement a simple and cheap approach, we decided to use one smartphone only in the clinical trials. Further researches might investigate on the use of two or more devices, although dealing with severe issues as time synchronization and sensor mismatch among smartphones. Consequently, we are not able to provide a full representation of the gait cycle, which is represented in Fig. 2, since one sensor node is not able to achieve this. Rather, our aim is to evaluate some additional features that could be used for a possible home-based lower limb rehabilitation, by focusing on the features in Table 1 that should be easy to recover using the adopted hardware setup.

The proposed system for gait monitoring is illustrated in the flow chart of Fig. 3 and it can be summarized into several main operations, described in the following subsections.

\subsubsection{Raw data collection from sensors}

Firstly, data acquisition is performed as follows:

- the user opens the application (we worked in this case on an Android ${ }^{\circledR}$ device but any operating system can be adopted) and sets the recording time;

- the smartphone is put into the band's pocket and fasten around the calf;

- the user taps the "Start" command on the screen and, after a countdown of $3 \mathrm{~s}$, the app begins to record data;

- during the recording time, the user performs the walking test and a device's vibration will advise him/her that recording is terminated;

- at the end of the trial, the user uploads data into the database after explicit consensus granted via the application.

If the user is a voluntary hemiplegic patient, an assistant helps him/her in any demands. Accelerometer, gyroscope, and magnetometer data are captured during each trial. However, in the following we will not consider magnetometer data because they are too sensitive to the presence of metal objects in the environment.

\subsubsection{Data resampling}

Once data are collected from sensors, the second main step consists in resampling them in order to reduce the

Fig. 2 The general gait cycle

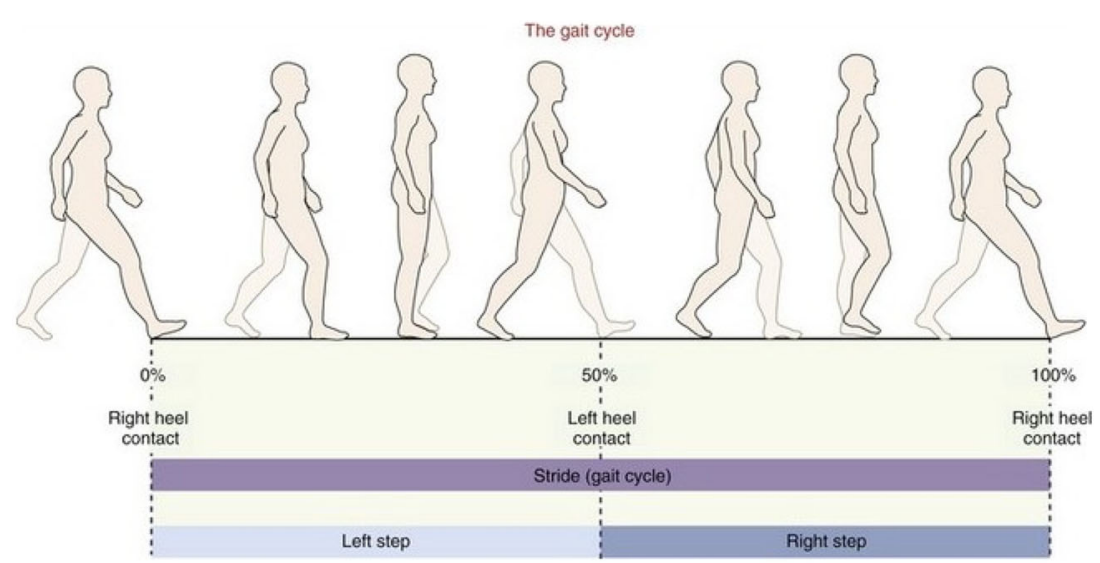


Table 1 Spatio-temporal features adopted for the gait analysis

\begin{tabular}{lll}
\hline Feature & Unit & Description \\
\hline Mean cycle duration & $\mathrm{s}$ & Mean value of the stride duration during a trial \\
Cycle regularity & $\mathrm{s}$ & Standard deviation of the stride duration during a trial \\
Cadence & $\mathrm{step} / \mathrm{min}$ & Number of strides executed in a given time \\
PSD peak acceleration & $\mathrm{dB}$ & Maximum value of the acceleration's PSD \\
PSD peak gyroscope & $\mathrm{dB}$ & Maximum value of the gyroscope's PSD
\end{tabular}

differences caused by the fact that acquisition is carried out using different smartphones with heterogeneous control hardware and sensor technologies. In addition, the app code usually cannot set the sampling rate at software level, as it depends on the hardware available on the adopted device. Depending on which device is used, each sensor data is sampled at a different rate (i.e., from 50 to $350 \mathrm{~Hz}$ ). In order to apply the same denoising algorithm to the whole set of data, we need a common sampling rate. Since $200 \mathrm{~Hz}$ is a reasonable trade-off, also considering the final target rate after wavelet filtering discussed successively, depending on the starting rate we did resampling or low-pass filtering to obtain the same rate.

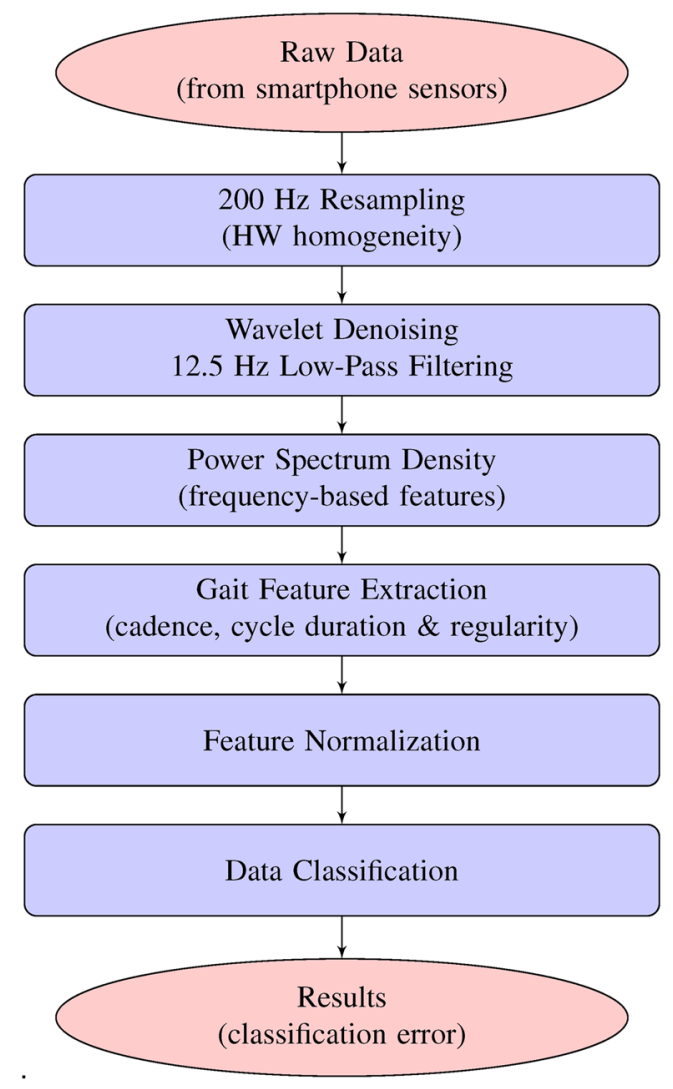

Fig. 3 Flow chart of the proposed processing system

\subsubsection{Denoising and filtering}

The successive step consists in denoising both acceleration and gyroscope data by a wavelet-based estimation algorithm and, successively, in low-pass filtering the reconstructed signals in order to make easier the feature extraction process.

A wavelet-based denoising algorithm is firstly applied by using the following model [28]:

$s(n)=f(n)+\sigma e(n)$,

where $n$ is the sample (time) index, $s(n)$ is the noisy signal, $f(n)$ is the signal to be recovered, $e(n)$ is a zero-mean, unitvariance Gaussian white noise and $\sigma$ is the noise level. The adopted algorithm is able to suppress the noise part of the signal $s(n)$ and to recover $f(n)$ through the following steps:

1. a wavelet decomposition of $s(n)$ at level $W$ is evaluated (we used the family of Daubechies' least asymmetric wavelets as default option);

2. a thresholding operation is performed to detail coefficients for each computed level from 1 to $W$;

3 . the wavelet reconstruction is computed based on the level $W$ original approximation coefficients and on the modified detail coefficients from level 1 to $W$.

In the following, we will consider $W=4$ levels taking into account a minimum of 128 samples per data recording trial. Noise estimate is performed at each wavelet level to scale the reference noise model $\sigma=1$, then a soft thresholding is performed by using a "universal threshold" approach for minimax performance [29], using a threshold proportional to $\sqrt{2 \ln (L)}$ where $L$ is the length of the considered signal.

After denoising, acceleration and gyroscope signals are passed through a 4-level Mallat's filter bank [30] for lowpass filtering and downsampling. In fact, average walking frequency of healthy people is about $1.8 \mathrm{~Hz}$ [31] and hence, as shown in Fig. 4, a final sampling frequency of $12.5 \mathrm{~Hz}$ is suitable to make easier the subsequent feature extraction process without loosing any useful information. 
Fig. 4 An example of denoising and filtering on the absolute value of the acceleration signal
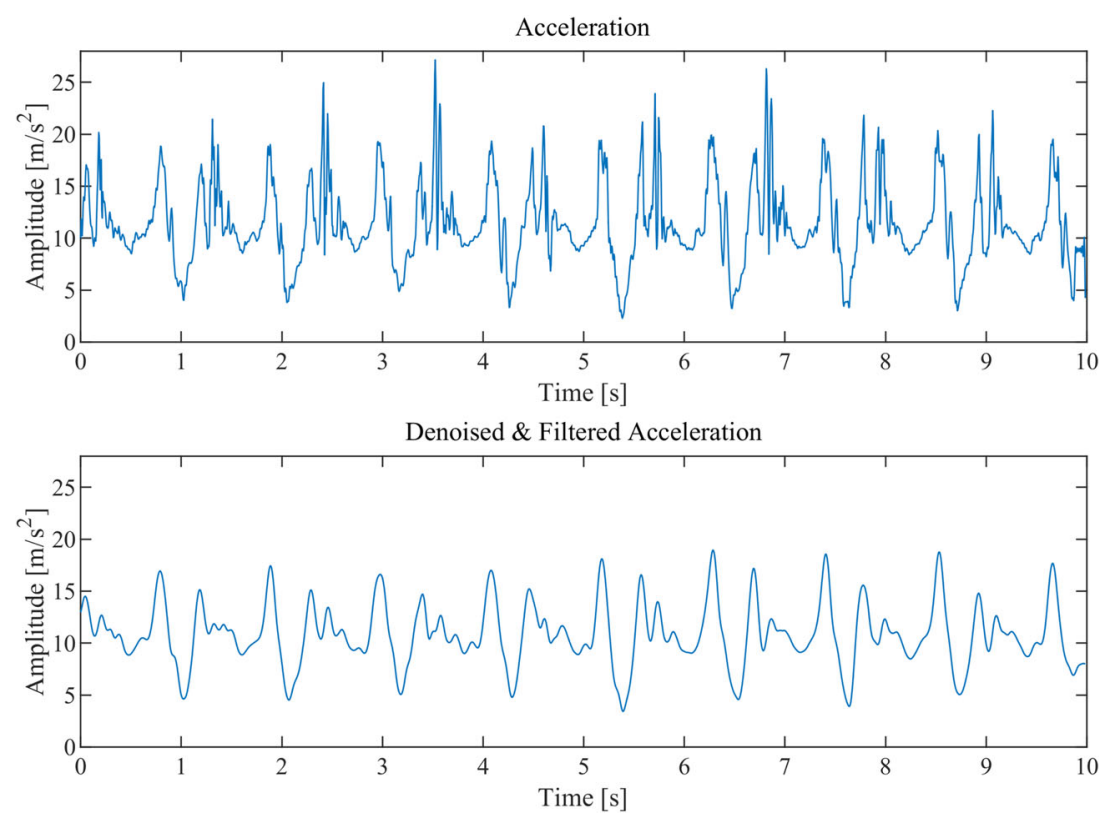

\subsubsection{Power spectrum density estimation}

As a well-known result achieved in the literature [32], the feature extraction for gait classification analysis should be based also on the power spectrum density (PSD) of considered signals, which coincide with the absolute values of acceleration and gyroscope signals after denoising and filtering. PSD estimation is then performed by the "Periodogram" method and we used the maximum PSD magnitude values (i.e., peaks) for both acceleration and gyroscope data. An example in this regard is shown in Fig. 5, where input denoised unfiltered data are shown.

It is worth to point out that the average stride frequency of every stride can be estimated and used as a parameter to find the walking cycle in each trial; in most cases, the acceleration's average frequency (PSD peak) is located at the gyroscope's second harmonic (see Fig. 5). However, because of relevant fluctuations due to the mechanism through which data are measured, this rule could not be always satisfied.

\subsubsection{Gait feature extraction}

In addition to the PSD peaks introduced before, other three features are used for gait cycles discrimination: cycle duration $\left(C_{d}\right)$, cycle regularity $\left(C_{r}\right)$, and cadence or revolutions per minute $\left(R_{m}\right)$.

Those features are generally used by the clinicians, in combination with other information on the subjects, to
Fig. 5 An example of PSD extraction and peak detection on denoised acceleration and gyroscope data at the original sampling rate of $200 \mathrm{~Hz}$
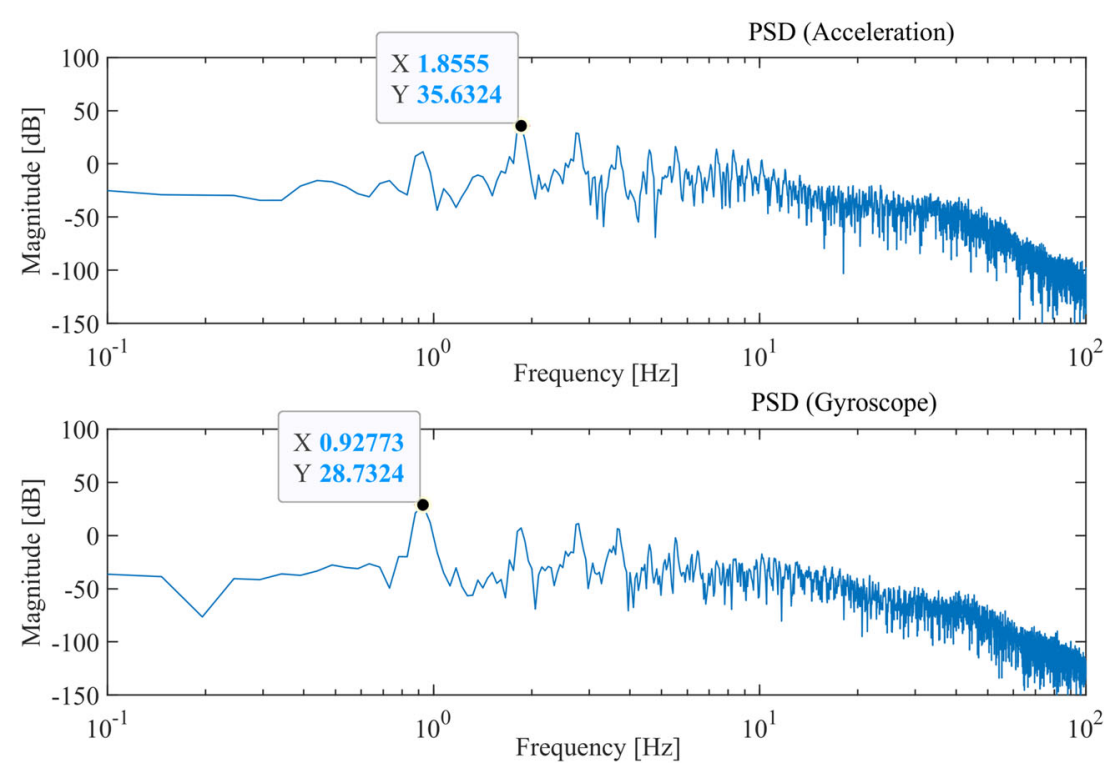
monitor the progress of a therapy or the evolution of a disease [5, 33, 34]. By the proposed approach, we propose a novel use of these features through the synergy of extracting gait features by low-cost devices and making classification automatically by means of machine learning models.

Each stride is recognizable from the acceleration pattern as the time between two "valleys". In fact, when the foot hits the ground, the sudden acceleration causes a spike followed by a deceleration that is represented by a valley; then, the successive leg swinging causes a new acceleration and the process is repeated cyclically. However, this behavior is more evident in healthy people rather than hemiplegic ones, as shown in Fig. 6.

In order to find out the time instants of foot contacts, both accelerometer and gyroscope data are considered. First, the absolute PSD's peak of both accelerometer and gyroscope data is measured; from the related frequency we can obtain a gross estimate of the average time between two steps considering either acceleration or gyroscope data, respectively. The average of these time intervals is then used as a rolling window to find a minimum in a small interval around an initial guess in accelerometer data, the final result is shown in Fig. 6.

Since we assume that the first valley is the starting point of the first stride and that one stride is performed during the time gap between two consecutive valleys, we can define the cycle (stride) duration as:

$C_{d}[k]=V[k+1]-V[k]$,

where $V[k]$ is the array containing the valleys' locations (in seconds) and $k$ is their index. So, $C_{d}[k]$ will represent the time difference between two valleys of a gait cycle. The cycle (stride) regularity is expressed as the standard deviation of the elements in the vector $C_{d}$, it is measured in second and proves regularity when the value of $C_{r}$ tends to zero. Finally, the cadence is defined as:

$R_{m}=\frac{60 N_{c}}{V_{l}-V_{f}}$

where $N_{c}$ is the number of cycles taken from recorded array, $V_{l}$ is the last element of the valley location (in seconds) and $V_{f}$ is the first one. Consequently, the cadence is the projection of how many strides could be performed in a minute and it is thus expressed in cycles/min.

All of the features adopted in this paper are summarized in Table 1; for the cycle duration representing a recorded trial we consider the average value of the elements in the vector $C_{d}$.
Fig. 6 An example of stride recognition using the absolute value of the time acceleration pattern for a healthy and unhealthy user
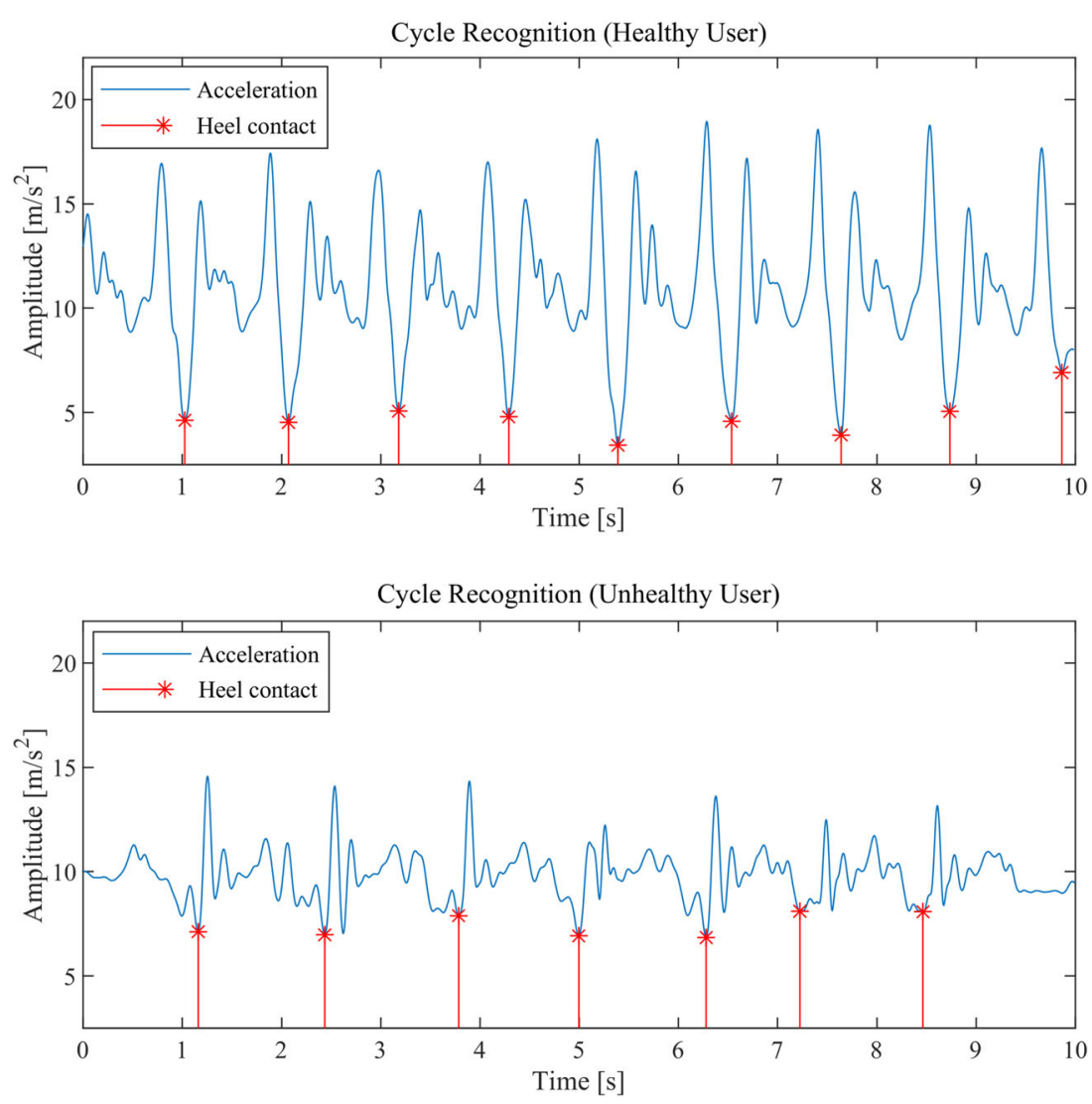


\subsubsection{Feature normalization}

Before using data for classification purposes it is helpful to perform data normalization in order to scale the features in the same numerical range, which in this case is chosen between 0 and 1 . Let $M$ be the number of patterns in the available dataset, where each pattern $\mathbf{x}_{m}, m=1 \ldots M$, is a collection of $N$ features (i.e., $N=5$ in the present approach) associated with a specific recorded trial:

$\mathbf{x}_{m}=\left[x_{m_{1}} x_{m_{2}} \ldots x_{m_{N}}\right], m=1 \ldots M$.

Since data features are completely heterogeneous, patterns cannot be normalized globally but with different affine transformations of features independent from one another:

$x_{m_{j}} \leftarrow \frac{x_{m_{j}}-b_{j}}{a_{j}-b_{j}}, j=1 \ldots N, m=1 \ldots M$,

where the terms are defined as $a_{j}=\max _{m}\left\{x_{m_{j}}\right\}$ and $b_{j}=\min _{m}\left\{x_{m_{j}}\right\}$, with $j=1 \ldots N$.

\subsubsection{Data classification and results}

The last step of the proposed algorithm consists in training a binary classifier by using well-known machine learning paradigms in order to categorize data and discriminate between healthy and unhealthy people. This is useful also to understand if the considered gait features are able to support this kind of classification, as for many other application fields [35-38].

We have investigated in our experiments all of the possible combinations of input features, therefore considering $2^{5}-1=31$ different datasets. A 10 -fold stratified validation is performed and several classification algorithms are compared in terms of classification error for each dataset.

\subsection{Statistics}

Several classification algorithms are used to assess the validity of the proposed approach:

- Linear Discriminant Analysis (LDA): tries to characterize data using a linear polynomial in order to separate patterns into two or more classes. It maximizes the inter-class discriminatory information by using the Fisher Discriminant technique for surface separation $[39,40]$. For the method to perform well data should satisfy the homoscedastic hypothesis, no hyperparameters are to be set in advance.

- Quadratic Discriminant Analysis (QDA): similarly to the LDA, tries to characterize a dataset using a quadratic polynomial based on Gaussian density conditional functions [41, 42]. It does not require any assumption on data, so it is more suitable for real contexts, no hyperparameters are to be set in advance.

- K-Nearest Neighbor (KNN): classifies a pattern depending on the most frequent class in the neighborhood of the pattern itself [43]. It does not require any assumptions on data and, in the following, we will use the Euclidean distance between patterns and $K=5$ as a default value.

- Naive Bayes (NB): is a statistical technique that seeks to verify if an element belongs to a class based on Bayes' Theorem [44, 45]. The algorithm calculates various conditional probabilities and assigns the patterns to the class with the highest probability. In the following we will use Gaussian kernel smoothing to estimate and model the data density.

- Support Vector Machine (SVM): is a particular supervised learning approach that can be applied for both regression and classification problems [46, 47]. Based on the solution of a quadratic convex problem, it is used for finding global minimum also in nonlinear complex problems. In the following we will adopt as default options a Radial Basis Function (RBF) kernel with Sequential Minimal Optimization (SMO) solver.

- Neuro-Fuzzy classifier (NF): is used to partition the feature space into fuzzy sets and assign non mutually exclusive membership values representing the reliability of the pattern of belonging to each class [48, 49]. In the following the model will be trained by a scaled conjugate gradient method with 100 maximum epochs and one cluster per class.

- Classification and Regression Tree (CART): operates by recursively splitting data until ending points, defined by some predefined criteria, are achieved [50, 51]. It should handle with nonlinear relations between features and classes [52], finding a correct trade-off among computational complexity and accuracy. Prior class probabilities will be estimated in the following based on class frequencies.

- Probabilistic Neural Network (PNN): this approach is based on a four-layer neural network employing Bayesian decision-making theory and data-driven learning $[53,54]$. The spread of radial basis functions will be set by default to 0.1 .

- Fuzzy Inference System (FIS): this method adopts first-order fuzzy rules and a data-driven inference system trained by means of the Substractive Clustering method [48, 55]. Gaussian membership functions will be adopted with one rule per fuzzy cluster and 0.5 influence of the cluster center (normalized data space).

All the classifiers use the same set of data, no ad hoc changes are made to make every dataset suited for the specific classification model. It can be underlined, however, 
Fig. 7 Maximum PSD magnitude of acceleration and gyroscope of healthy (blue) and unhealthy (red) subjects
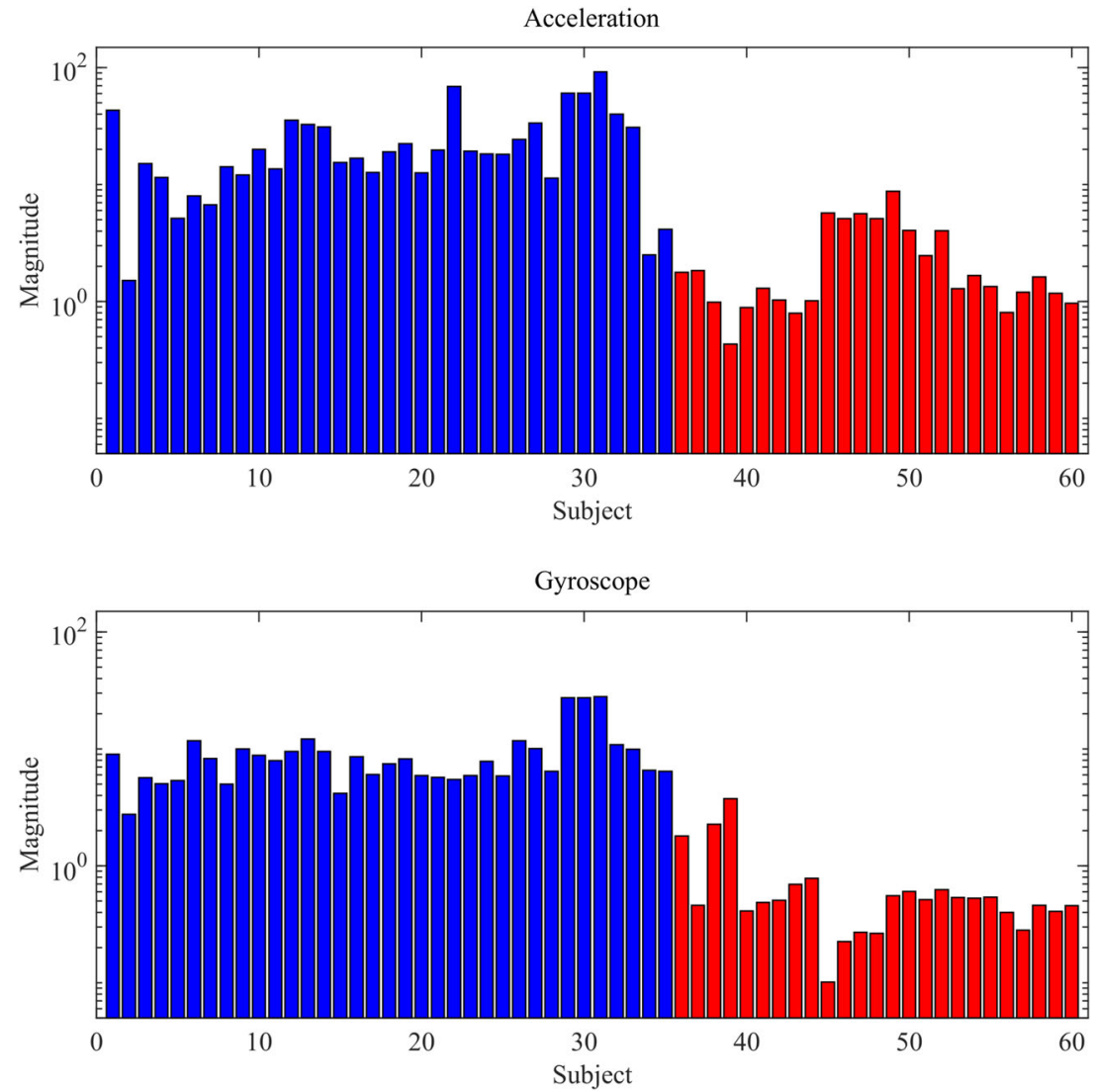

that some general differences exist in the way by which each algorithm extract information from the data. For instance, KNN and SVM classifiers do not provide from training data a mathematical model of the classifier. In fact, SVM classifier gives as output the support vectors, while KNN seeks, for each pattern to be classified, the nearest patterns which the output label is extracted from.

On the contrary, statistical and fuzzy logic-based classification algorithms aim at finding, by using training data, the parameters of a mathematical model that is able to infer the probability of or the fuzzy membership to a class, respectively, for the pattern under classification. An intermediate behavior is the one of CART classifiers, where a decision tree is obtained by training data rather than a parametric model. Further details can be found in the references cited for each classifier listed above.

\section{Results}

In this section we report the obtained numerical results. For the sake of illustration, let us consider firstly the PSD magnitude of acceleration and gyroscope for healthy classification. Looking at Fig. 7, where the first 35 (blue) records are from the healthy group while the successive 25 (red) records are of the post-stroke patients, by the differences of the maximum PSD magnitude the reader could have a sufficient but not so accurate estimation of the

Table 2 Best feature subset per classifier and number of times a feature is adopted

\begin{tabular}{|c|c|c|c|c|c|c|c|c|c|c|}
\hline Feature & LDA & QDA & $\mathrm{KNN}$ & NB & SVM & NF & CART & PNN & FIS & Occurrences \\
\hline Mean cycle duration & 0 & 1 & 1 & 0 & 0 & 1 & 0 & 0 & 0 & 3 \\
\hline Cycle regularity & 0 & 0 & 0 & 1 & 0 & 0 & 0 & 0 & 0 & 1 \\
\hline Cadence & 0 & 1 & 0 & 0 & 1 & 0 & 0 & 1 & 0 & 3 \\
\hline PSD peak acceleration & 0 & 0 & 1 & 0 & 0 & 0 & 0 & 0 & 0 & 1 \\
\hline PSD peak gyroscope & 1 & 1 & 1 & 1 & 1 & 1 & 1 & 1 & 1 & 9 \\
\hline
\end{tabular}


healthy status. Consequently, a more accurate classification approach is required in order to perform a robust analysis.

To this end, we considered the classification models introduced in Section 2.3 and we performed an exhaustive search considering all the 31 sets of possible combinations of the 5 features listed in Table 1. A stratified 10fold validation procedure was adopted for evaluating the classification accuracy; for each subset of features a classifier is trained 6 times, by classifying each time 10 different patterns (i.e., subjects or recorded trials) and using the remaining 50 patterns for training. As most of the classification models depend upon a random initialization of model parameters, and the 10-fold partitioning of the dataset is random as well, we repeated the above procedure 10 times and the ones considered in the following are the average values of accuracy obtained over the 10 different trials.

For each classifier we report in Table 2 the subset of features that yielded the best (average) classification accuracy, among all of the possible combination of features. More precisely, there are 5 rows (one per feature) and 10 columns (one per each algorithm plus the number of times that each feature is selected in an optimal dataset); each element of the table takes value 1 if the corresponding feature is selected and 0 otherwise. In case of identical values we chose the subset with the lower number of features according to a regularization approach [56].

The numerical classification results are summarized in Table 3 where, for each classification algorithm, there are reported the average classification accuracy and the related standard deviation over the 10 trials carried out in correspondence of the subset of features that yielded the best (average) accuracy. The number of adopted features is obtained by summing the ones in the related column of Table 2.

\section{Discussion}

By analyzing the results obtained in Table 2, it is evident that the acceleration is not a useful feature while the gyroscope must be taken into account for a good discrimination. In fact, the sole use of the PSD Peak Gyroscope feature is the best option for LDA, CART, and FIS classifiers. On the other hand, QDA, SVM, and PNN are able to achieve a good classification by using some other features, such as Cadence, which is sufficient for SVM and PNN. It is worth to point out that these results are quite in accordance with current medical practices.

Looking at the overall performance of the proposed classification approaches, we note that the accuracy in discriminating among pathological and physiological gait is always maintained at high levels, from 80 to $90 \%$. In addition to PSD peak gyroscope, cadence is the feature that by means of PNN is able to obtain the best accuracy of $91.13 \%$. Cycle regularity allows NB classifier to achieve a $90.38 \%$, which the second score in the ranking. In all cases, the performance volatility measured by the standard deviation is adequate. Overall, the great performance of PNN with only 2 features does suggest that a data-driven machine learning approach can bring improvements with respect to statistical approaches based, for instance, on Discriminant Analysis and to non-parametric models as KNN as well.

As a final remark, we note that the previous numerical results are strictly dependent on the uncertainty of measures through which data are gathered and then processed. In the present case, error in measurements depends by two main factors: accuracy and precision of hardware sensors; objectivity of the experimental setup, mainly depending on the application of the smartphone on a same point of the body as well as on the reproducibility of clinical trials (same walking, same movements, etc.). In this work, the influence of such errors is mitigated by the use of several and different hardware devices and by the adoption of a relatively large number of patients during the clinical tests.

\section{Conclusions}

The novelty with respect to state-of-the-art applications is the combination of data acquisition and filtering on the
Table 3 Average classification accuracy and standard deviation for the best feature subset

\begin{tabular}{llll}
\hline Classifier & Average accuracy $(\%)$ & Std. deviation $(\%)$ & Adopted features \\
\hline LDA & 83.23 & \pm 1.28 & 1 \\
QDA & 88.39 & \pm 1.35 & 3 \\
KNN & 87.47 & \pm 1.25 & 3 \\
NB & 90.38 & \pm 0.89 & 2 \\
SVM & 89.84 & \pm 1.38 & 2 \\
NF & 89.41 & \pm 1.12 & 2 \\
CART & 87.74 & \pm 1.07 & 1 \\
PNN & 91.13 & \pm 1.12 & 2 \\
FIS & 86.40 & \pm 1.19 & 1 \\
\hline
\end{tabular}


device, with pattern recognition and data fusion techniques that provide a correct discrimination of gait movements. An exhaustive feature selection approach is considered in order to find out the best subset of features able to discriminate among healthy and unhealthy subjects.

The procedure has been used also for evaluating the performance of several classification models in terms of classification accuracy. Very good performances, even achieving a $100 \%$ of accuracy, are obtained on the clinical trials performed in this research. It is important to point out that this is a feasibility study, not a clinical trial of a model. However, the results are very promising for making possible to assist medical specialists in analyzing the rehabilitation path in the near future.

In particular, the model could be extended for using it in specific and personalized programs for home rehabilitation meant to improve the patient's quality of the life while boosting the treatment effectiveness and thus shortening the patient's recovery time.

Funding Open Access funding provided by Università degli Studi di Roma "La Sapienza".

Open Access This article is licensed under a Creative Commons Attribution 4.0 International License, which permits use, sharing, adaptation, distribution and reproduction in any medium or format, as long as you give appropriate credit to the original author(s) and the source, provide a link to the Creative Commons licence, and indicate if changes were made. The images or other third party material in this article are included in the article's Creative Commons licence, unless indicated otherwise in a credit line to the material. If material is not included in the article's Creative Commons licence and your intended use is not permitted by statutory regulation or exceeds the permitted use, you will need to obtain permission directly from the copyright holder. To view a copy of this licence, visit http:// creativecommonshorg/licenses/by/4.0/.

\section{References}

1. Nilsson NJ (1969) Survey of pattern recognition. Ann N Y Acad Sci 161(2):380-401

2. Secco J, Farina M, Demarchi D, Corinto F, Gilli M (2016) Memristor cellular automata for image pattern recognition and clinical applications. In: Circuits and Systems (ISCAS), 2016 IEEE International Symposium on. IEEE, pp 1378-1381

3. Altilio R, Liparulo L, Panella M, Proietti A, Paoloni M (2015) Multimedia and gaming technologies for telerehabilitation of motor disabilities [leading edge]. IEEE Technol Soc Mag 34(4):23-30

4. Pugazhenthi D, Priya VS (2013) Pattern recognition using automatic image classification and recognition methods: A literature review. International Journal of Engineering Sciences \& Research Technology, pp 1354-1356

5. Whittle MW (2014) Gait analysis: an introduction. ButterworthHeinemann

6. Tao D, Li X, Wu X, Maybank SJ (2007) General tensor discriminant analysis and gabor features for gait recognition. IEEE Trans Pattern Anal Mach Intell 29(10)
7. Altilio R, Paoloni M, Panella M (2017) Selection of clinical features for pattern recognition applied to gait analysis. Med Biol Eng Comput 55(4):685-695

8. Horst F, Kramer F, Schäfer B, Eekhoff A, Hegen P, Nigg BM, Schöllhorn WI (2016) Daily changes of individual gait patterns identified by means of support vector machines. Gait Posture 49:309-314

9. Liparulo L, Zhang Z, Panella M, Gu X, Fang Q (2017) A novel fuzzy approach for automatic Brunnstrom stage classification using surface electromyography. Med Biol Eng Comput 55(8):1367-1378

10. Samuel OW, Li X, Fang P, Li G (2016) Examining the effect of subjects' mobility on upper-limb motion identification based on emg-pattern recognition. In: Asia-Pacific Conference on Intelligent Robot Systems (ACIRS). IEEE, pp 137-141

11. Cesqui B, Tropea P, Micera S, Krebs HI (2013) Emg-based pattern recognition approach in post stroke robot-aided rehabilitation: a feasibility study. J Neuroeng Rehab 10(1):75

12. Dipietro L, Ferraro M, Palazzolo JJ, Krebs HI, Volpe BT, Hogan N (2005) Customized interactive robotic treatment for stroke: Emgtriggered therapy. IEEE Trans Neural Syst Rehab Eng 13(3):325334

13. Shusong $X, X i a ~ Z ~(2010)$ Emg-driven computer game for poststroke rehabilitation. In: 2010 IEEE Conference on Robotics Automation and Mechatronics (RAM). IEEE, pp 32-36

14. Zhang X, Zhou P (2012) High-density myoelectric pattern recognition toward improved stroke rehabilitation. IEEE Trans Biomed Eng 59(6):1649-1657

15. Mantyjarvi J, Lindholm M, Vildjiounaite E, Makela S-M, Ailisto HA (2005) Identifying users of portable devices from gait pattern with accelerometers. In: 2005. Proceedings.(ICASSP'05). IEEE International Conference on Acoustics, Speech, and Signal Processing, vol 2. IEEE, pp ii-973

16. Thang HM, Viet VQ, Thuc ND, Choi D (2012) Gait identification using accelerometer on mobile phone. In: 2012 International Conference on Control, Automation and Information Sciences (ICCAIS). IEEE, pp 344-348

17. Zhang Z, Liparulo L, Panella M, Gu X, Fang Q (2016) A fuzzy kernel motion classifier for autonomous stroke rehabilitation. IEEE J Biomed Health Inf 20(3):893-901

18. Li J, Besada JA, Bernardos AM, Tarrío P, Casar JR (2017) A novel system for object pose estimation using fused vision and inertial data. Inf Fusion 33:15-28

19. Niyogi SA, Adelson EH et al (1994) Analyzing and recognizing walking figures in xyt. In: CVPR, vol 94, pp 469-474

20. Qin L, Ma H, Liao W-H (2015) Insole plantar pressure systems in the gait analysis of post-stroke rehabilitation. In: 2015 IEEE International Conference on Information and Automation. IEEE, pp 1784-1789

21. Zhang Z, Fang Q, Ferry F (2011) Upper limb motion capturing and classification for unsupervised stroke rehabilitation. In: IECON 2011-37th Annual Conference on IEEE Industrial Electronics Society. IEEE, pp 3832-3836

22. DelRosario MB, Redmond SJ, Lovell NH (2015) Tracking the evolution of smartphone sensing for monitoring human movement. Sensors 15(8):18901-18933

23. LeMoyne R, Mastroianni T, Cozza M, Coroian C, Grundfest W (2010) Implementation of an iPhone as a wireless accelerometer for quantifying gait characteristics. In: 2010 Annual International Conference of the IEEE Engineering in Medicine and Biology. IEEE, pp 3847-3851

24. Nishiguchi S, Yamada M, Nagai K, Mori S, Kajiwara Y, Sonoda T, Yoshimura K, Yoshitomi H, Ito H, Okamoto K et al (2012) Reliability and validity of gait analysis by android-based smartphone. Telemed e-Health 18(4):292-296

25. LeMoyne R, Mastroianni T (2017) Wearable and wireless gait analysis platforms: smartphones and portable media devices. 
In: Wireless MEMS Networks and Applications. Elsevier, pp 129152

26. Yamada M, Aoyama T, Mori S, Nishiguchi S, Okamoto K, Ito T, Muto S, Ishihara T, Yoshitomi H, Ito H (2012) Objective assessment of abnormal gait in patients with rheumatoid arthritis using a smartphone. Rheumatol Int 32(12):3869-3874

27. Ellis RJ, Ng YS, Zhu S, Tan DM, Anderson B, Schlaug G, Wang Y (2015) A validated smartphone-based assessment of gait and gait variability in Parkinson's disease. PLoS one 10(10):e0141694

28. Abramovich F, Benjamini Y, Donoho DL, Johnstone IM (2006) Adapting to unknown sparsity by controlling the false discovery rate. Ann Stat 34(2):584-653

29. Donoho DL, Johnstone IM (1994) Ideal spatial adaptation by wavelet shrinkage. Biometrika 81(3):425-455

30. Mallat SG (1989) A theory for multiresolution signal decomposition: The wavelet representation. IEEE Trans Pattern Anal Mach Intell 11(7):674-693

31. Ji T et al (2005) Frequency and velocity of people walking. Struct Eng 84(3):36-40

32. Preece SJ, Goulermas JY, Kenney LPJ, Howard D (2009) A comparison of feature extraction methods for the classification of dynamic activities from accelerometer data. IEEE Trans Biomed Eng 56(3):871-879

33. Perry J, Davids JR, etal. (1992) Gait analysis: normal and pathological function. J Pediatr Orthop 12(6):815

34. Vaughan CL, Davis BL, O'Connor JC (1992) Dynamics of human gait. Human Kinetics Publishers

35. Dash M, Liu H (1997) Feature selection for classification. Intell Data Anal 1(1):131-156

36. Rizzi A, Buccino NM, Panella M, Uncini A (2008) Genre classification of compressed audio data. In: 2008 IEEE 10th Workshop on Multimedia Signal Processing, pp 654-659

37. James G, Witten D, Hastie T, Tibshirani R (2013) An introduction to statistical learning. Springer

38. Proietti A, Liparulo L, Leccese F, Panella M (2016) Shapes classification of dust deposition using fuzzy kernel-based approaches. Measurement 77:344-350

39. Fisher RA (1936) The use of multiple measurements in taxonomic problems. Ann Eugenics 7:179-188

40. Balakrishnama S, Ganapathiraju A (1998) Linear discriminant analysis-a brief tutorial. Inst Signal Inf Process 18

41. Srivastava S, Gupta MR, Frigyik BA (2007) Bayesian quadratic discriminant analysis. J Mach Learn Res 8:1277-1305

42. Hastie T, Tibshirani R, Friedman J (2009) The elements of statistical learning: Data mining, inference, and prediction, 2nd edn. Springer

43. Cover T, Hart P (1967) Nearest neighbor pattern classification. IEEE Trans Inf Theory 13(1):21-27

44. Manning CD, Raghavan P, Schütze M (2008) Introduction to information retrieval. Cambridge University Press

45. Rish I (2001) An empirical study of the naive bayes classifier. In: IJCAI 2001 workshop on empirical methods in artificial intelligence, vol 3. IBM New York, pp 41-46

46. Cortes C, Vapnik V (1995) Support-vector networks. Mach Learn 20(3):273-297

47. Suykens JohanAK, Vandewalle J (1999) Least squares support vector machine classifiers. Neural Process Lett 9(3):293-300

48. Sun C-T, Jang J-S (1993) A neuro-fuzzy classifier and its applications. In: 1993., Second IEEE International Conference on Fuzzy Systems. IEEE, pp 94-98

49. Cetişli B, Barkana A (2009) Speeding up the scaled conjugate gradient algorithm and its application in neuro-fuzzy classifier training. Soft Comput 14(4):365-378

50. Breiman L, Friedman J, Olshen R, Stone C (1984) Classification and regression trees. CRC Press
51. Rokach L, Maimon O (2008) Data mining with decision trees: theory and applications. World Scientific Pub Co Inc.

52. Friedl MA, Brodley CE (1997) Decision tree classification of land cover from remotely sensed data. Remote Sens Environ 61(3):399-409

53. Specht DF (1990) Probabilistic neural networks. Neural Netw 3(1):109-118

54. Wasserman PD (1993) Advanced methods in neural computing. Van Nostrand Reinhold

55. Chiu S (1994) Fuzzy model identification based on cluster estimation. J Intell Fuzzy Syst 2(3):267-278

56. Bishop CM (1995) Neural networks for pattern recognition. Oxford University Press

Publisher's note Springer Nature remains neutral with regard to jurisdictional claims in published maps and institutional affiliations.

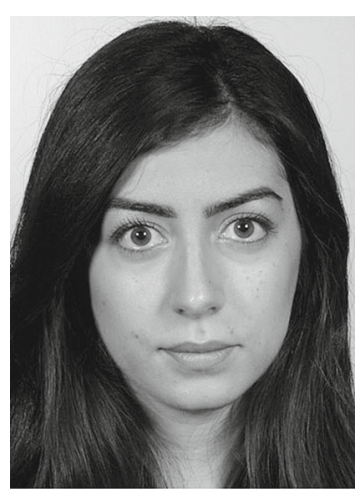

Rosa Altilio is a Research Fellow in Information and Communication Engineering and she received the Ph.D. with Honors in this field at the University of Rome "La Sapienza" in 2017.

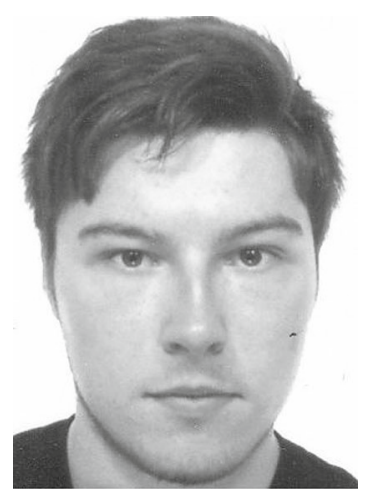

Andrea Rossetti received the Bachelor and Master Degree in Electronic Engineering both with Honors at University of Rome "La Sapienza" in 2014 and in 2017 , respectively. $\mathrm{He}$ is currently working as a HWSW engineer for an international Company.

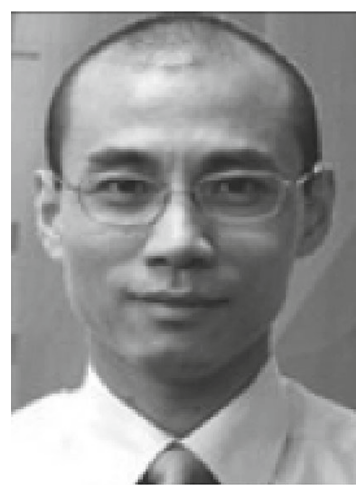

Qiang Fang is a Professor, Chair of the Department of Biomedical Engineering at Shantou University, China. His research focuses on biomedical signal processing and bioinstrumentation. 


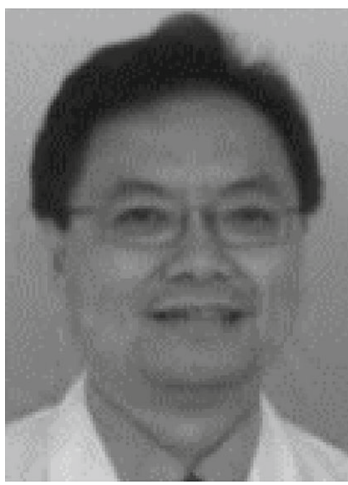

Xudong $\mathrm{Gu}$ is the chairman of the Rehabilitation Centre at Jiaxing 2nd Hospital, a professor at Jiaxing University, China, and a standing committee member of Chinese Medical Doctor Association, Rehabilitation Doctor Association.

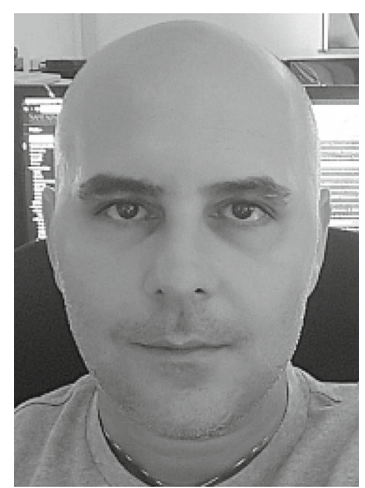

Massimo Panella received in 2002 the Ph.D. degree in Information and Communication Engineering from the University of Rome "La Sapienza", where he is currently Associate Professor of Electrical Engineering and Machine Learning. 